\title{
PLANETARY NEBULAE AND NITROGEN ENRICHMENT IN THE GALAXY
}

\author{
D.C.V.Mallik \\ Indian Institute of Astrophysics, Bangalore.
}

\begin{abstract}
All stars in the mass interval $1-4 \mathrm{M}_{0}$ probably evolve through a double shell source phase before losing their entire envelopes through stellar wind and the ejection of planetary nebula shells. The remant carbon-oxygen core rapidly evolves to a blue nucleus, illuminates the surrounding nebula for a few tens of thousands of years and finally cools off as a white dwarf (Paczynski, 1970, Harm and Schwarzschild 1975). A large fraction of the mass contained in the main-sequence stars of mass $1-4 \mathrm{MO}_{0}$ thus returns to the interstellar medium. A common feature of the double she11 source (DSS) evolution of stars is the occurrence of He shell flashes. Evolutionary studies by Schwarzschild and Harm (1967), Iben (1975) and others show that the flash-driven convection zone carries helium burning products towards the hydrogen-rich layers. The consequences of mixing between the outer convective envelope and the intershell region have received a great deal of attention in recent years particularly in connection with the interpretation of carbon stars. If a deep temporary convection zone exists extending from the surface of the star to a point near the helium burning shell and mixing is allowed to take place for sufficiently long time Sackmann, Smith gnd Despain (1974) found that the flash-produced ${ }^{12} \mathrm{c}$ underwent further owo processing enriching the surface of the star in 14 . Iben (1976) has also speculated on the passibility of surface enrichment of ${ }^{14} \mathrm{~N}$ during the DSS evolution of intermediatemass stars. The subsequent loss of this envelope as a planetary nebula shell can thus cause nitrogen enrichment of the interstellar gas. In the present work we have evaluated the extent of this enrichment and have also derived the gradient of nitrogen abundance in the disc of the Galaxy based on the simple model of galactic evolution due to Talbot and Arnett (1973, he reinafter TA).
\end{abstract}


stars in the DSS phase obey a we l determined core mass-luminosity relationship (Paczynski 1970) and since the hydrogen shell remains the dominant energy source between shell flashes, the rate of core growth mas be expressed as a function of the core mass. Integration of this relation yields the DSS lifetime of a star,once the core masses at the beginning and the end of the DSS phase are known for the star. The DSS lifetime of stars of different parent masses was derived with the help of the models of Wood and Cahn (1977). Further, the relation between the interflash period and the core mass (Paczynski 1975) was used to determine the maximum possible number of flashes for a star of a particular mass. Fur the purpose of the present calculation it was sufficient to use a mean interflash period for each star. Wood and Cahn (1977) have obtained a distribution of planetary nebula masses and of the remnant white dwarf masses assuming that all low mass stars ( $1 \mathrm{M}_{0} \leqslant M \leqslant 4 \mathrm{M}_{0}$ ) evolve through a typical Mira variable phase before ejecting a planetary nebula shell and leaving a white dwarf remnant. Since they have included in their model the effect of massloss via stellar wind prior to planetary nebula ejection it is also possible to determine the initial and final envelope masses for each star during the DSS Phase.

The change in the surface abundance of ${ }^{12} \mathrm{C}$ due to shell flashes has been calculated by Iben (1976) for a $7 \mathrm{M}_{0}$ star with a core mass of $0.95 \mathrm{M}_{0}$. If $\mathrm{n}_{12}$ denote the abundance by number of ${ }^{12} \mathrm{C}$ and $n_{16}$ the same for 160 , the ration

$$
\frac{n_{12}}{n_{16}}=0.4+\frac{1.5}{g} \frac{N}{N_{\max }} \quad \ldots(1),
$$

where $\mathrm{g}$ is the mean mass fraction of the stellar envelope during the DSS evolution, $N$ the number of shell flashes and $\mathrm{N}_{\max }$ the maximum possible number of flashes. For a star of lower mass, equation (1) is modified by multiplying the right side into $\frac{1.4-M_{c, i}}{0.45}$ where $M_{g i}$ is the core mass at the beginning of the DSS evolution. Since the abundance of $16_{0}$ hardly changes through shell flashing, we have assumed that $n_{16}$ equals its initial value. The number $N$ is arbitrary and in the present work we have set $N=100$. Since the surface enrichment is linear in $N$ it is trivial to calculate the same for any other value of $N$. Finally we have assumed that the extra ${ }^{12} \mathrm{C}$ produced through shell flashes is entirely converted to ${ }^{14} \mathrm{~N}$ in the deep mixing phase through CNO processing. This extreme assumption does provide an upper 
limit to the primary production of ${ }^{14} \mathrm{~N}$ in low mass stars. The number ratios were converted to mass ratios and the amount of additional ${ }^{14} \mathrm{~N}$ produced was calculated as a function of the parent stellar mass. To be specific, we have considered the additional $14 \mathbb{N}$ produced and mixed in the planetary nebula itself.

To calculate the yield for a generation of stars we adopted the IMF specified by TA,

$$
\Psi_{m}=\zeta(\mu-1) m^{-\mu}
$$

with $\xi=0.25$ and $\mu=1.55$. The production matrix for the generation was calculated from

$$
q_{i j}=\int_{m} \Psi_{m} Q_{i j}(m) d m
$$

where $Q_{i j}$ is the fraction of the mass of the star originally in the form of species $j$ which is eventually ejected as species $i$. The production matrix is given in Table 1. The elements $q_{N /}$ and $q_{N 4}$ correspond to the pri=

\begin{tabular}{|c|c|c|c|c|c|c|}
\hline & & Table & 1 & & & \\
\hline \multirow{2}{*}{$\underset{i}{\text { Product }}$} & \multicolumn{6}{|c|}{ Progenitor $i$} \\
\hline & $T$ & 4 & $\mathrm{CO}$ & $\mathbf{N}$ & $n r$ & h \\
\hline $\begin{array}{r}1 \\
4 \\
\mathrm{CO} \\
\mathrm{N} \\
\mathrm{nr} \\
\mathrm{h}\end{array}$ & $\begin{array}{l}0.16 \\
1.09(-2) \\
7.1(-3) \\
4.87(-4) \\
2.4(-3)\end{array}$ & $\begin{array}{l}0.17 \\
7.1(-3) \\
4.87(-4) \\
2.4(-3)\end{array}$ & $\begin{array}{l}0.13 \\
4.09 \\
9.4\end{array}\left(\begin{array}{l}-2 \\
-3\end{array}\right)$ & $\begin{array}{l}0.17 \\
9.4(-3)\end{array}$ & 0.18 & 0.18 \\
\hline
\end{tabular}
mary production of ${ }^{14} \mathrm{~N}$ as calculated in the present work.

To obtain the distribution of nitrogen in the disc of the galaxy we followed the simple TA model which assumes (i) instantaneous recycling of the gas, (ii) a time invariant IMF and (iii) no mass inflow. In this case we have for a species $i$ with abundance $X i$,

$$
\frac{d x_{i}}{d y}=p_{i}-\Lambda_{i} x_{i}
$$

$y=\ln \left[\frac{M_{\text {gas }}(0)}{M_{\text {gas }}(t)}\right], \quad p_{i}=\sum_{i \neq j} \frac{q_{i j} x_{j}}{1-f}, \Lambda_{i}=\frac{f-q_{i i}}{1-f}$.

Here $\mathbf{P}$ is the total fraction of mass returned per generation. 
Equation (3) was solved for ${ }^{14} \mathrm{~N}$ and $16_{0}$ as a function of $\mathrm{y}$. Gordon and Burton (1976) have derived a radial distributioil of interstellar gas in the disc from observations of co. The co densities have been used with certain assumptions to obtain $\mathrm{H}_{2}$ densities coupled with a mass model of the galaxy due to Innanen (1966); this distribution yields values of $y$ at various distances $R$ from the centre of the Galaxy. A plot of the nitrogen and oxygen abundances as a function of $R$ is shown in Figure 1 .

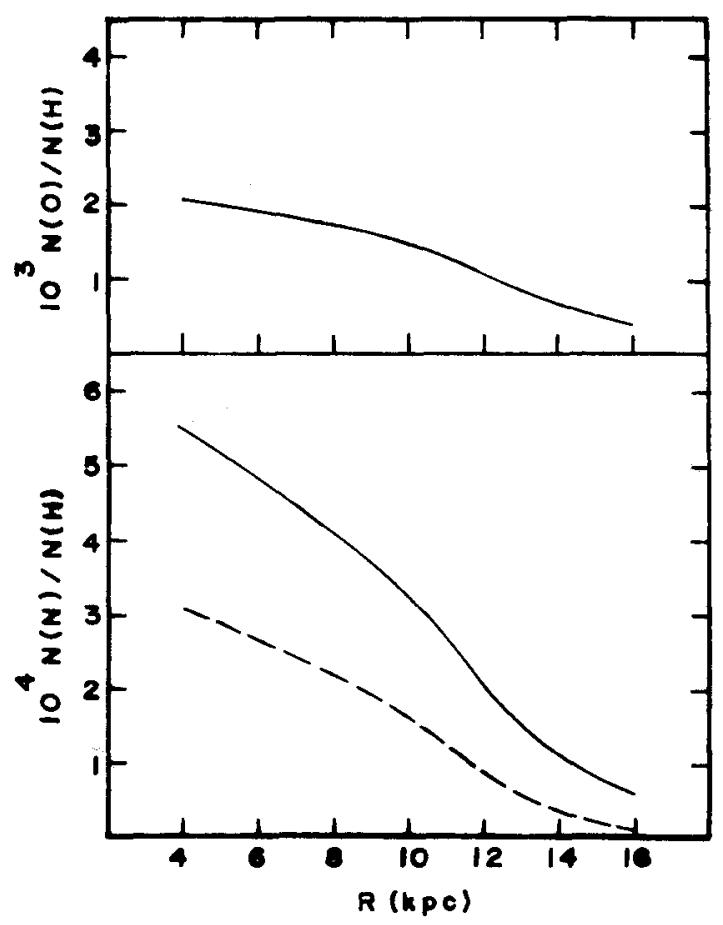

Figure 1. Nitrogen and oxygen abundances by number as a function of the distance. The dashed line in the lower half corresponds to ${ }^{14} \mathrm{~N}$ distribution without primary production.

It is seen that the primary production of ${ }^{14} \mathrm{~N}$ in low 
mass stars contributes nearly $50 \%$ to the total abundance of ${ }^{14} \mathrm{~N}$ in the galaxy. The abundance gradients in the solar neighbourhood are found to be $\frac{d \log n}{n}=-0.18$ and -0.13 for nitrogen and oxygen respectively. The nitrogen gradient agrees well with the observed value due to Peimbert (this colloquium). The oxygen gradient is steeper than the observed value. The gradients are sensitive to the distribution of the interstellar gas and any readjustment of the $\mathrm{H}_{2}$ radial distribution will reflect on them.

References.

Gordon, M.A. and Burton W.B.: 1976, Astrophys.J.208, 346. Harm, R. and Schwarzschi 1d,M.: 1975, Astrophys.J.200,324. Iben, I.,Jr: 1975, Astrophys.J., 196,525.

Iben, I.,Jr: 1976, Astrophys.J., 208,165.

Innanen, K.A.; 1966, Z.Astrophys.64, 158 .

Paczynski,B.: 1970, Act.Astr.20,47.

Paczynski,B .: 1975, Astrophy s.J., 202, 558 .

Sackmann,I.J.,Smith,R.L. and Despain,K.H.: 1974, Astrophys. J. 187,555 .

Schwarzschild,M. and Harm,R.: 1967, Astrophys.J.,150,961. Talbot,R.J. and Arnett,W.D.: 1973, Astrophy s.J.186,51.

Wood,P.R. and Cahn, J.H.: 1977, Astrophy s.J.211, $\overline{499}$. 